\section{La enfermedad de Alzheimer en el año $2000^{1}$}

Palabras clave: enfermedad de Alzheimer, envejecimiento, demencia.

\footnotetext{
Basado en el documento "2000 progress report on Alzheimer's disease. Taking the next steps", elaborado por el Instituto Nacional de Envejecimiento (National Institute on Aging) de los Estados Unidos de América. Disponible en versión electrónica en http://www. alzheimers.org, y en versión impresa en: Alzheimer's Disease Education and Referral (ADEAR) Center. PO Box 8250. Silver Spring. MD 20907-8250. USA
}

La enfermedad de Alzheimer (EA) es una enfermedad neurodegenerativa cerebral progresiva, irreversible y relacionada con el envejecimiento, que produce pérdida de la memoria, deterioro intelectual y trastornos de la personalidad y del comportamiento. Su evolución es variable, pero, por término medio, la supervivencia tras el diagnóstico es de 8 a 10 años. En la mayoría de los casos los síntomas aparecen después de los 60 años. La EA es la causa más importante de demencia en mayores de 65 años y en los Estados Unidos de América (EE. UU.) constituye un importante problema de salud pública por sus repercusiones en los pacientes, sus familias, el sistema de salud y la sociedad en general. En este país se estima que puede haber 4 millones de afectados y la prevalencia de la enfermedad se duplica cada 5 años a partir de los 65. La incidencia es de aproximadamente 360000 casos al año y aumentará a medida que la población envejezca. La EA representa una importante carga económica para la sociedad. En los EE. UU. se calcula que los costos anuales directos e indirectos de la atención a los afectados pueden llegar a los cien mil millones de dólares.

Todo esto hace que la investigación sobre la EA sea prioritaria, en particular si se tiene en cuenta que una intervención que retrasara su inicio en un promedio de 5 años reduciría a la mitad el número de pacientes en el año 2050. La investigación sobre la EA financiada por el gobierno federal de los EE. UU. está dividida en tres grandes áreas: 1) causas y factores de riesgo, 2) diagnóstico, y 3) tratamiento y atención a los pacientes. En este informe se describen las investigaciones realizadas en estos campos durante el año 2000 con el apoyo del Instituto Nacional de Envejecimiento (National Institute on Aging: NIA) y de otros organismos dependientes de los Institutos Nacionales de la Salud (National Institutes of Health: NIH) de los EE. UU.

\section{ENFERMEDAD DE ALZHEIMER}

Durante mucho tiempo se creyó que la EA y los síntomas de demencia eran una consecuencia inevitable del envejecimiento, pero hoy se sabe que no es así. Durante el último cuarto de siglo se han hecho importantes adelantos en la investigación de la etiología, diagnóstico y tratamiento de la enfermedad, que al mismo tiempo han ampliado los conocimientos sobre las enfermedades neurodegenerativas rela- 
cionadas con la edad, la función cerebral en ancianos sanos y la forma de reducir el deterioro cognitivo normal relacionado con el envejecimiento. Inicialmente, la EA destruye las neuronas de regiones cerebrales implicadas en el control de la memoria, como el hipocampo. Más tarde, afecta a la corteza cerebral, y en particular a las zonas responsables del lenguaje y el razonamiento, pudiendo producir también cambios de personalidad. Finalmente, se ven afectadas muchas otras regiones cerebrales, que se atrofian, y el paciente se vuelve incontinente, queda confinado en la cama y deja de responder al mundo exterior. En cambio, el envejecimiento normal no se asocia con la pérdida de un gran número de células cerebrales.

\section{Alteraciones estructurales}

La EA se caracteriza por dos alteraciones estructurales principales: las placas amiloides y las redes neurofibrillares.

Placas amiloides. Aparecen primero en las regiones cerebrales implicadas en la memoria y en otras funciones cognitivas. Consisten en depósitos extracelulares insolubles de amiloide $\beta$, que es el producto de la fragmentación de una proteína mayor, denominada proteína precursora del amiloide (APP). Aunque todavía no se sabe si las placas son causa o consecuencia de la enfermedad, hay pruebas de que la formación de depósitos de amiloide puede ser un proceso fundamental. De hecho, la modificación de la estructura de la APP puede causar al menos una forma hereditaria de la enfermedad, caracterizada por mutaciones del gen de esta proteína. Estudios recientes han proporcionado mucha información sobre la naturaleza del amiloide $\beta$ y su toxicidad neuronal, sobre la formación de las placas y su depósito en el cerebro y sobre la forma de reducir su número.

Redes neurofibrillares. En las neuronas normales, los microtúbulos adoptan una disposición en vía de tren, mantenida por la proteína tau, que actúa como los durmientes. En la EA la proteína tau está alterada y dos moléculas se enrollan helicoidalmente, una alrededor de la otra. La agregación de estos filamentos da lugar a la formación de las redes neurofibrilares y a la separación de los microtúbulos, con la consiguiente alteración del mecanismo de transporte intracelular.

\section{Etiología}

La EA es una enfermedad de etiología multifactorial, en la que intervienen factores genéticos y ambientales.
Factores genéticos. Existen dos tipos de EA: familiar y esporádica. La EA puede clasificarse también en función de la edad de inicio: precoz, en menores de 65 años, o tardío, en mayores de 65 años. La EA de inicio precoz es rara ( 5 a $10 \%$ de los casos), generalmente afecta a personas de 30 a 60 años y suele progresar más rápidamente que la de inicio tardío. Algunas formas de EA de inicio precoz son hereditarias. Todas las formas conocidas hasta ahora de EA familiar tienen inicio precoz y en más de la mitad de los casos son causadas por mutaciones autosómicas dominantes de tres genes localizados en cromosomas distintos: el gen de la APP en el cromosoma 21, el gen de la presenilina 1 (PS1) en el cromosoma 14 y el gen de la presenilina 2 (PS2) en el cromosoma 1. Aunque el número total de casos debidos a estas causas es muy pequeño (100 a 200 en todo el mundo) y no hay pruebas de que estas mutaciones estén implicadas en la EA esporádica de inicio tardío, mucho más frecuente, esto no significa que los factores genéticos no tengan una participación importante en la EA no familiar.

Así, por ejemplo, hay una relación entre los alelos del gen de la apolipoproteína $\mathrm{E}(A P O E)$ y el riesgo de EA de inicio tardío. La ApoE participa en el transporte del colesterol sanguíneo y está presente en las neuronas y en las células gliales normales. En las placas amiloides su cantidad está muy aumentada. El alelo ع2, relativamente raro, podría proteger a algunas personas frente a la EA, mientras que el alelo $\varepsilon 4$ incrementa el riesgo. Es más, cuanto mayor es el número de alelos $\varepsilon 4$, menor es la edad de inicio de la enfermedad. Por su parte, el alelo ع3, el más frecuente en la población en general, parece tener un papel neutral en la enfermedad. De cualquier modo, no hay que olvidar que algunas personas pueden tener uno o ambos alelos $\varepsilon 4 \sin$ padecer la enfermedad y que otras pueden padecerla sin tener ninguno. Por consiguiente, el alelo $\varepsilon 4$ constituye un factor de riesgo de EA, pero no su causa. Todavía no se sabe cómo contribuye la ApoE $\varepsilon 4$ al aumento del riesgo de EA, pero entre los posibles mecanismos se encuentran la facilitación de la acumulación del amiloide $\beta$ en las placas, la interacción con las concentraciones de colesterol y los efectos sobre la muerte de las neuronas. Numerosos estudios indican que podrían existir otros factores de riesgo genéticos.

Envejecimiento. Es el principal factor de riesgo de EA. En el curso del envejecimiento normal, el cerebro sufre numerosas alteraciones: mueren las neuronas de algunas regiones cerebrales, aunque no la mayoría de las neuronas importantes para el aprendizaje; se atrofian algunas neuronas y sus conexiones, especialmente en regiones importantes para el aprendizaje, la memoria, la planificación 
y otras actividades mentales complejas; aparecen redes neurofibrilares y placas amiloides en algunas regiones cerebrales; aumenta la susceptibilidad de las mitocondrias a las lesiones, y aumentan la inflamación y la carga oxidativa. En individuos sanos, el impacto de estas alteraciones puede ser ligero, produciendo diferentes grados de pérdida de la memoria, pero en los pacientes con EA algunas de ellas son mucho más pronunciadas. Se están realizando numerosos estudios sobre los procesos implicados en el envejecimiento normal del cerebro con el fin de conocer mejor sus diferencias con lo que ocurre en la EA. Entre los aspectos más investigados se encuentran los mecanismos oxidativos y la posible participación de las cardiopatías o la hipertensión. En este sentido, se sabe que las enfermedades cerebrovasculares constituyen la segunda causa más frecuente de demencia y hay indicios de que los infartos cerebrales y la EA podrían estar relacionados. Otro factor potencialmente importante es la concentración de colesterol, cuya elevación podría aumentar la velocidad de depósito de las placas. Por último, se están desvelando muchos paralelismos entre la EA y otras enfermedades neurodegenerativas progresivas que causan demencia, como las enfermedades de Parkinson (EP) y Huntington y las enfermedades por priones. Además del depósito de proteínas anormales en el cerebro, todas ellas comparten otras características importantes.

\section{Diagnóstico}

En la actualidad, el diagnóstico clínico de la "EA posible" (demencia que también podría deberse a alguna otra causa, como los accidentes cerebrovasculares) y la "EA probable" (demencia en la que no se puede encontrar ninguna otra causa) en pacientes con problemas de memoria o de otras funciones mentales está basado, sobre todo, en la anamnesis, el examen físico y diferentes pruebas neuropsicológicas que miden la memoria, la capacidad lingüística y otras funciones cerebrales. Los especialistas pueden diagnosticar clínicamente la enfermedad con una exactitud del $90 \%$, pero la única forma de establecer el diagnóstico de certeza sigue siendo el examen neuropatológico del cerebro tras la autopsia, para comprobar si la acumulación de placas amiloides y redes neurofibrilares en determinadas regiones cerebrales es característica de la enfermedad.

Cuanto antes se establezca el diagnóstico clínico de la enfermedad, mayores son las probabilidades de éxito del tratamiento sintomático durante más tiempo. Un área que está siendo objeto de intensas investigaciones es la del deterioro cognitivo leve (individuos con problemas de memoria que no cumplen los criterios clínicos de EA), puesto que el $40 \%$ de los afectados acaban padeciendo la enfermedad en un plazo de 3 años. El conocimiento de las características diferenciales y del curso clínico de la EA y del deterioro cognitivo leve sería fundamental para ayudar al médico a establecer un diagnóstico temprano y exacto de la EA.

Las pruebas radiológicas son útiles para descartar tumores y accidentes cerebrovasculares. En los últimos años se han producido importantes adelantos en este campo, con la aparición de nuevas técnicas, como la resonancia magnética, la tomografía de emisión de positrones o la tomografía computadorizada de emisión unifotónica. Todas ellas están siendo utilizadas sobre todo como herramientas de investigación, pero, junto con otras medidas diagnósticas, son muy prometedoras como forma de llegar a conseguir una identificación más precoz de las personas en riesgo de sufrir EA.

\section{Tratamiento}

El objetivo final es encontrar tratamientos que atajen los procesos fundamentales de la EA e impidan su progresión hacia un estado de deterioro de la función cognitiva y de la calidad de vida, pero para quienes ya padecen la enfermedad, lo más urgente es disponer de tratamientos que controlen los síntomas. Dichos tratamientos deberían ser eficaces en diferentes tipos de pacientes, mantener su eficacia a lo largo del tiempo, mejorar una amplia gama de síntomas, la función cognitiva y la capacidad para realizar las tareas de la vida cotidiana y estar exentos de efectos colaterales importantes. La Administración de Alimentos y Medicamentos (Food and Drug Administration: FDA) de los EE.UU. ha aprobado tres medicaciones para la EA: tacrina, donepezilo y rivastigmina. Todas ellas actúan inhibiendo la acetilcolinesterasa, pero ninguna revierte ni detiene la enfermedad, y su eficacia es de duración limitada.

\section{INVESTIGACIÓN EN EL AÑO 2000}

En el año 2000, las investigaciones sobre la EA apoyadas por el NIA y otros organismos de los NIH han posibilitado importantes adelantos en diferentes áreas.

\section{Etiología}

Amiloide. Aunque no hay pruebas formales de que el bloqueo de la producción o depósito de amiloide $\beta$ prevenga la enfermedad, este ha sido uno de los cen- 
tros de atención de la investigación. En el informe de 1999 (1999 progress report on Alzheimer's disease) se describieron los primeros pasos para desarrollar una vacuna antiamiloide en ratones transgénicos portadores de formas mutantes humanas de la APP; su administración repetida generó una respuesta inmunitaria que eliminó casi completamente la formación de placas amiloides. En el año 2000 se han obtenido nuevos resultados alentadores. Se comprobó que la vacuna no es tóxica en diferentes animales de experimentación y los estudios preliminares de seguridad en humanos revelaron que una vacunación es bien tolerada. Si se confirma su seguridad en el ser humano, el fabricante tiene proyectado iniciar los estudios de eficacia en el año 2001. También se ha realizado un estudio sobre la administración intranasal de la misma vacuna en ratones. Aunque esta vía de administración no fue tan eficaz como la inyección, tendría la ventaja de ser mejor tolerada a largo plazo.

Otros investigadores están explorando los mecanismos naturales mediante los cuales algunas células microgliales destruyen el amiloide y han comprobado que algunos componentes del sistema de complemento reducen la captación de amiloide $\beta$ por estas células. Por consiguiente, los mecanismos que inhiben el proceso inflamatorio podrían incrementar la capacidad de la microglía para destruir el amiloide.

Una forma de evitar la formación de placas consiste en detener la producción de amiloide $\beta$, objetivo que se puede conseguir por diferentes vías. Por ejemplo, se ha verificado que los estrógenos y la testosterona pueden reducir la secreción de amiloide $\beta$ por neuronas cultivadas, pero todavía queda por comprobar que este efecto también ocurre in vivo y que es de magnitud suficiente para reducir la acumulación de amiloide. Otra forma de evitar la formación de placas es la degradación del amiloide $\beta$ antes de que se agregue en placas insolubles. La enzima de degradación de la insulina puede ejercer este efecto in vitro. Otro método consiste en crear pequeños péptidos que impiden que el amiloide $\beta$ forme placas.

Presenilinas. La PS1 podría ser una de las enzimas que rompen la APP y originan la producción de amiloide $\beta$. Además, las presenilinas tienen muchas otras funciones, entre las que se encuentra su participación en la apoptosis, y también en la comunicación intercelular, a través del mantenimiento de las sinapsis. Sin embargo, todavía queda por determinar cuál es la función que, una vez alterada en pacientes con mutaciones de la presenilina, inicia la enfermedad.

Apoptosis. La EA se caracteriza por la muerte anormal de neuronas vulnerables en determinadas regiones cerebrales, lo cual ha suscitado un gran interés por la apoptosis. En este sentido, se ha comprobado que el amiloide $\beta$ puede inducir la apoptosis mediada por las caspasas. Por consiguiente, cabe la posibilidad de obtener tratamientos farmacológicos o génicos eficaces dirigidos frente a las caspasas implicadas en este proceso de muerte celular asociado con el amiloide $\beta$. Otra diana terapéutica podría ser la telomerasa, enzima que mantiene la estructura cromosómica. En determinadas condiciones, la telomerasa puede bloquear algunas vías apoptósicas específicas y reducir la vulnerabilidad de las células cultivadas a la muerte inducida por el amiloide $\beta$. Sin embargo, todavía está por ver si esto también ocurre in vivo.

ApoE y otros vínculos genéticos. En ratones transgénicos, la sustitución de su gen APOE por el alelo humano $\varepsilon 4$ se asocia con mayores depósitos de amiloide y con mayores cantidades de amiloide $\beta$ fibrilar que su sustitución por el alelo $\varepsilon 3$. Además, se han identificado otros genes o regiones cromosómicas asociadas con la predisposición a la EA de inicio tardío. Una de estas regiones está situada en el cromosoma 12; aunque todavía no se ha identificado exactamente el gen o genes implicados, uno de ellos podría ser el de la $\alpha_{2}$-macroglobulina. Los estudios de población también han señalado posibles factores de riesgo, como un polimorfismo del gen de la interleuquina-1 que multiplica por dos el riesgo de EA. Asimismo, hay estudios que señalan la posible existencia de factores genéticos que protegerían a determinados individuos particularmente longevos frente al deterioro intelectual. No obstante, hay que tener en cuenta que muchas de las asociaciones genéticas identificadas hasta ahora podrían ser espurias.

Agregación de proteínas en otras enfermedades neurodegenerativas. Una característica común de las enfermedades neurodegenerativas es la agregación anormal de proteínas en el cerebro: amiloide en la EA, sinucleína en la EP, huntingtina en la enfermedad de Huntington, priones en las enfermedades por priones, BRI en la demencia familiar británica (FBD) y proteína tau en las taupatías como la demencia frontotemporal con EP (FTDP-17), la parálisis supranuclear progresiva o la enfermedad de Pick. La mayoría de los investigadores creen que los agregados de proteínas que se forman en estas enfermedades son tóxicos y que quizás se pudiera detener la enfermedad si se consiguiera encontrar formas de evitar la agregación o los procesos puestos en marcha por ella.

La participación de la proteína tau en estos procesos ha sido investigada en dos modelos de ratones transgénicos. En uno de ellos, con hiperproducción de una de las seis formas de la proteína tau, 
la agregación de esta llevaba a la pérdida de los microtúbulos de las neuronas y a la degeneración de los axones, alteraciones similares a las observadas en la FTDP-17 y en el complejo esclerosis lateral amiotrófica-parkinsonismo-demencia de la isla de Guam. En el otro, tras la introducción de un gen de la proteína tau humana que contenía la mutación más frecuente en la FTDP-17, los animales presentaron alteraciones de la marcha, de otros movimientos y del comportamiento, encontrándose una relación directa entre el grado de expresión del gen mutante, el número de redes neurofibrilares y la edad.

La sinucleína $\alpha$ es una proteína que se acumula de forma anormal en las placas de la EA, en los cuerpos de Lewy de la EP y de la enfermedad por cuerpos de Lewy, y en un grupo de enfermedades denominadas sinucleinopatías. Una forma rara de EP hereditaria se debe a una mutación del gen de la sinucleína $\alpha$, y la proteína mutante forma depósitos insolubles. La acumulación presináptica de la proteína interfiere con la liberación de los neurotransmisores y puede interrumpir los circuitos neuronales. En el año 2000, las funciones de la sinucleína $\alpha$ han sido investigadas en tres modelos. En el primero, in vitro, la proteína solo se observó en el lado presináptico de la sinapsis, y solo después de la formación de las conexiones interneuronales, lo cual indica que podría tener una función reguladora en la sinapsis; sin embargo, queda por averiguar si la alteración cualitativa o cuantitativa de la sinucleína $\alpha$ modifica la cantidad de neurotransmisor que pasa de una neurona a la otra. En otro modelo, los ratones transgénicos con sobreexpresión de la proteína padecían alteraciones de la función motora similares a las observadas en la EP. En un tercer modelo, los ratones transgénicos con sobreexpresión de una forma anormal de un tipo de APP presentaban un gran número de prolongaciones neuronales que contenían sinucleína en localizaciones muy similares a las observadas en la enfermedad por cuerpos de Lewy. Estos datos señalan una vez más la existencia de relaciones entre la EA, la enfermedad por cuerpos de Lewy y la EP.

Las enfermedades por priones, entre las que se incluye la enfermedad de Creutzfeldt-Jakob de los humanos, son causadas por el plegamiento anormal de un prión. Al contrario de estas enfermedades, la EA no es infecciosa, pero tiene muchos paralelismos con ellas, como el hecho de que tanto los priones como el amiloide $\beta$ forman estructuras amiloideas en el cerebro.

Además de la EA, existen otras demencias asociadas con defectos genéticos que conducen a la formación de otros tipos de depósitos de amiloide en el cerebro. Entre ellas está la FBD, que suele manifestarse entre los 40 y los 50 años, que se caracte- riza por demencia progresiva, parálisis y pérdida del equilibrio, y en la que existe un defecto del gen $B R I$, situado en el cromosoma 13. Al igual que en la EA, los pacientes presentan depósitos de amiloide y redes neurofibrilares. La mutación del gen $B R I$ hace que se produzcan formas de la proteína más largas que la normal y parte de la nueva proteína es cortada y se deposita en el cerebro en forma de placas amiloides que producen disfunción neuronal y demencia. También se ha demostrado la participación de una proteasa denominada furina en la formación de las fibrillas. En el año 2000 se identificó en una pequeña población danesa una variante de la FBD caracterizada por acumulación de amiloide alrededor de los vasos sanguíneos, cataratas, sordera, pérdida de equilibrio y demencia. El defecto consiste en la mutación de una única base en el mismo gen implicado en la FBD. Una vez más, la mutación da lugar a la formación de una proteína más larga que la habitual, cuyo corte da lugar a la producción del amiloide.

Un posible tratamiento para evitar la agregación de proteínas consiste en el empleo de las denominadas chaperonas, proteínas que hacen que otras proteínas se plieguen normalmente y no se agreguen. Un grupo de investigadores ha introducido en Caenorrhabditis elegans el segmento de proteína anormal que da lugar a la enfermedad de Huntington, junto con la chaperona Hsp 104. Cuando se expresaba solo el segmento de proteína anormal de la enfermedad de Huntington, se producía su agregación, que era tóxica para este nemátodo, mientras que cuando también se expresaba la chaperona Hsp 104 disminuía tanto la agregación como la toxicidad.

Edad. Durante el envejecimiento normal se producen algunos cambios en el funcionamiento cerebral y los investigadores están tratando de identificar las diferencias entre estos procesos y lo que ocurre en la EA. Así, por ejemplo, se ha comprobado que la pérdida de marcadores celulares de la remodelación neuronal es mucho más acentuada en la EA. Otro campo de investigación muy activo es el de la reducción de la producción de radicales libres, y en la actualidad se están realizando varios ensayos clínicos para determinar si el tratamiento con antioxidantes puede retrasar el deterioro cognitivo relacionado con el envejecimiento o la aparición de la EA.

Otros factores. Otra forma de investigar las causas de la enfermedad consiste en la realización de estudios epidemiológicos poblacionales, que han señalado ya varias posibilidades. Así, se ha verificado que la hipercolesterolemia constituye un factor de riesgo de EA en personas sin ningún alelo 
APOE \&4, pero no en aquellos con uno o dos de estos alelos. Otros estudios han indicado que el riesgo de EA se asocia a la coincidencia de una escolaridad de menos de 6 años con la residencia en el medio rural durante la infancia. Aunque los estudios epidemiológicos constituyen un valioso complemento de la investigación básica y pueden proporcionar resultados interesantes, sus datos también pueden ser contradictorios e incompletos, entre otros motivos debido a las diferencias metodológicas, a la complejidad de los problemas analizados y al gran número de variables implicadas.

\section{Diagnóstico}

El diagnóstico clínico de la EA ha mejorado de forma sustancial en los últimos años y siguen las investigaciones con el fin de encontrar formas fiables, válidas y sencillas de identificar la EA en sus fases iniciales. Además, hacen falta pruebas que permitan distinguir de forma fiable a los pacientes con EA de aquellos cuyos problemas cognitivos se deben a otras causas. Cuando existan tratamientos eficaces, el diagnóstico de la EA en sus fases iniciales posibilitará el inicio del tratamiento antes de que las alteraciones cerebrales produzcan alteraciones cognitivas.

Pruebas neurorradiológicas. Un estudio de resonancia magnética (RM) de las regiones implicadas en la memoria y en las funciones ejecutivas permitió identificar a las personas que más tarde padecerían la enfermedad. La RM proporcionó una exactitud del $100 \%$ para distinguir a los individuos sanos de los que ya tenían EA leve, y del 93\% para distinguir a aquellos de los que inicialmente presentaban alteraciones de la memoria y acabaron padeciendo EA. Sin embargo, no fue tan fácil distinguir a los que siguieron teniendo problemas de memoria sin progresar a la EA de aquellos que acabaron sufriendo la enfermedad. En otro estudio similar, se volvió a comprobar que existen varias regiones cerebrales específicas que pueden estar afectadas en la EA preclínica y que la atrofia de estas zonas puede señalar la ulterior progresión hacia la EA. En un estudio, el volumen del hipocampo se relacionó con la mayoría de las medidas de las funciones de adquisición y evocación a lo largo del espectro del envejecimiento normal hasta la EA. En un estudio se realizaron dos RM separadas por un intervalo de 12 meses en pacientes con EA y en individuos sanos; los resultados revelaron que la velocidad media de la atrofia cerebral era del $2,4 \%$ al año en los pacientes, frente al $0,4 \%$ en los controles. Esto demuestra que es posible utilizar la RM para cuantificar la atrofia cerebral a lo largo del tiempo y que su utilización en los ensayos clínicos podría permitir una monitorización de la EA a más corto plazo que las pruebas neuropsicológicas. Otros estudios preliminares han investigado posibles métodos de visualización de las placas. En uno, realizado in vitro, se utilizó la microscopia por RM y en el otro, in vivo, una sonda radiactiva.

Pruebas neuropsicológicas. También se están investigando formas de mejorar las pruebas neuropsicológicas relacionadas con funciones como la memoria o el lenguaje, con el fin de predecir mejor la aparición de la enfermedad.

Correlaciones clínico-patológicas. Otro importante foco de atención consiste en alcanzar una mejor comprensión de la relación entre las lesiones neuropatológicas iniciales y las manifestaciones clínicas. La estrecha relación entre la acumulación de placas seniles y redes neurofibrillares y la velocidad de deterioro clínico indican que estos marcadores tienen consecuencias clínicas y no son solo indicadores del envejecimiento. Asimismo, se ha observado que las concentraciones totales de dos tipos de péptidos amiloideos están elevadas en fases iniciales de la demencia y se correlacionan fuertemente con el deterioro cognitivo, lo cual apoya la importante participación del amiloide $\beta$ en las manifestaciones patológicas iniciales de la enfermedad.

Marcadores. La existencia de marcadores biológicos de la EA podría conducir a la identificación de las personas con EA preclínica. En este sentido, la pérdida inexplicada del olfato en presencia de uno o más alelos $A P O E \varepsilon 4$ se ha asociado con un alto riesgo de deterioro cognitivo. En otro estudio las concentraciones plasmáticas de amiloide $\beta$ fueron más elevadas en los individuos que posteriormente desarrollaron EA que en los que no lo hicieron.

\section{Tratamientos farmacológicos}

El mejor conocimiento de la neuroquímica y la neurobiología de la enfermedad ha originado una importante ampliación del número y tipos de fármacos que están siendo investigados. Se estima que el NIA, otros institutos de los NIH y diferentes laboratorios farmacéuticos están investigando entre 50 y 60 productos en ensayos clínicos. Estas investigaciones están centradas en los tratamientos de mantenimiento a corto plazo de la función cognitiva en pacientes con EA, en los tratamientos para reducir la velocidad de progresión de la enfermedad, retrasar su aparición o prevenirla totalmente, y en los tratamientos de los problemas conductuales relacionados con la EA. 
Mantenimiento a corto plazo de la función cognitiva. Se están estudiando o se han aprobado ya varios fármacos que mantienen temporalmente la función del sistema colinérgico. Los tres únicos fármacos aprobados hasta ahora por la FDA (tacrina, donepezilo y rivastigmina) pertenecen a esta clase, pero tienen el inconveniente de que no alteran el curso de la enfermedad.

Prevención de la enfermedad. En este aspecto, las investigaciones actuales están centradas en los estrógenos, los antiinflamatorios, los antioxidantes y los factores de crecimiento neuronal.

Múltiples estudios realizados en animales han indicado que los estrógenos tienen efectos positivos sobre la memoria. Los estudios epidemiológicos también han relacionado el consumo de estrógenos con una disminución del riesgo de EA. Además, se ha comprobado que tienen efectos antioxidantes y antiinflamatorios e incrementan el crecimiento de las prolongaciones de algunas neuronas. Sin embargo, los tres estudios clínicos realizados hasta ahora han indicado que el tratamiento de reposición de estrógenos no retrasa la progresión de la enfermedad ni mejora el pronóstico cognitivo y funcional. No obstante, estos tres estudios no han permitido contestar la pregunta fundamental: si las mujeres con envejecimiento normal que toman estrógenos tras la menopausia se ven protegidas frente a la aparición de la EA o frente al deterioro cognitivo relacionado con la edad. Ya están en marcha nuevos estudios para responder a esta pregunta.

Una de las características de la EA es la inflamación cerebral, pero no se sabe si esto es causa o consecuencia. De cualquier forma, los datos epidemiológicos indican que los antiinflamatorios, esteroideos y no esteroideos, se asocian con una disminución del riesgo de EA. Estudios recientes en ratones transgénicos también indican que los antiinflamatorios no esteroideos pueden reducir la producción de placas. Sin embargo, en un estudio clínico que comparó los efectos de la prednisona y el placebo no se detectaron diferencias entre los dos grupos con respecto al deterioro cognitivo. Antes de que se pueda llegar a una conclusión definitiva, son necesarios nuevos estudios en este campo.

La producción excesiva de radicales libres puede ocasionar lesiones celulares. Entre los antioxidantes más estudiados se encuentran las vitaminas C y E, el extracto de Ginkgo biloba, la melatonina, los flavonoides y los carotenoides. La vitamina $\mathrm{E}$ ya se está utilizando con fines terapéuticos, después de que un ensayo clínico revelara una disminución de la progresión de algunos aspectos clínicos de la EA a los 6 meses. En otro ensayo clínico se está investigando si el tratamiento precoz con vitamina $\mathrm{E}$ y do- nepezilo permite retrasar o evitar el comienzo de la EA. Asimismo se está investigando el extracto de Ginkgo biloba.

En un estudio realizado en monos se observó que la implantación en el cerebro de fibroblastos manipulados genéticamente para segregar factor de crecimiento nervioso evitaba la atrofia y la pérdida de las propiedades colinérgicas de las neuronas vecinas. Con base en estos resultados se han iniciado ya algunos estudios clínicos de fase I para investigar la seguridad de este tratamiento.

Tratamiento de los síntomas conductuales. Los síntomas conductuales son frecuentes en la EA y pueden ser graves. Ya existen varios fármacos para tratar estos síntomas, como los antidepresivos, los antipsicóticos y los sedantes, pero se siguen buscando nuevos tratamientos, farmacológicos y no farmacológicos. Un ensayo clínico sobre el tratamiento de los trastornos del sueño con melatonina está ya en la fase de análisis de los datos. Otro estudio ha investigado el tratamiento no farmacológico de la agitación, problema que afecta al 70 a $90 \%$ de los pacientes con EA y que puede dificultar mucho su permanencia en el domicilio. Los fármacos habitualmente utilizados ante este problema suelen ser eficaces, pero pueden tener importantes efectos colaterales.

Consideraciones sobre el futuro de la investigación clínica de la EA. El diseño y la realización de ensayos clínicos sobre posibles tratamientos de la EA plantea diversos problemas, como la necesidad de reclutar a un gran número de participantes, tanto pacientes con EA clínica y preclínica como ancianos normales, o la necesidad de tener en cuenta en el diseño las características especiales de esta población, cuya enfermedad hace que en muchas ocasiones los familiares y otras personas que cuidan de ellos tengan que participar activamente en los estudios. Asimismo, se plantean importantes problemas éticos, como el uso de pruebas genéticas que permiten identificar el riesgo de padecer la enfermedad.

\section{El apoyo a quienes cuidan a los pacientes}

Un poco más de la mitad de los pacientes son atendidos en el domicilio, y el resto en diferentes tipos de instituciones sanitarias. Un estudio reciente de más de 1500 personas que cuidaban a pacientes con EA reveló que, en comparación con quienes cuidan a otros enfermos, estas personas invierten mucho más tiempo en estas tareas y que su impacto es mucho mayor en diversos aspectos: consecuencias laborales, tensión, problemas de salud física y mental, tiempo de ocio y conflictos familia- 
res. Otras investigaciones han demostrado que las necesidades de estas personas cambian a lo largo del tiempo, a medida que la enfermedad evoluciona. Un estudio reciente reveló que los cuidadores con síntomas depresivos son incapaces de adaptarse y cumplir nuevas estrategias basadas en cambios de la conducta y modificaciones del medio físico. La investigación de la seguridad en el hogar es muy importante y un estudio reciente ha identificado seis problemas fundamentales: las lesiones por caída; las lesiones por ingestión de sustancias peligrosas; el perderse al salir de casa; las lesiones, propias o de otros, por objetos punzantes; el fuego y otras quemaduras, y la incapacidad para responder adecuadamente en situaciones de crisis.

En 1995, los NIH establecieron una importante iniciativa quinquenal, denominada $\mathrm{REACH}$ (Resources for Enhancing Alzheimer's Caregiver Health) ${ }^{2}$ para realizar investigaciones conductuales y sociales sobre intervenciones destinadas a ayudar a quienes cuidan de pacientes con EA y otras demencias. Dichas intervenciones han sido muy diversas: grupos de apoyo, programas de formación conductual, intervenciones familiares, modificaciones del medio y servicios informatizados de comunicación e información.

\section{Creación de una infraestructura de investigación}

Un importante componente del éxito de los NIH en la investigación sobre la EA ha sido su red de investigadores y centros de investigación. La creación y mantenimiento de esta infraestructura de investigación son un esfuerzo continuo que abarca aspectos tan diversos como el desarrollo de mecanismos innovadores de financiación de la investigación, el patrocinio de conferencias sobre temas de investigación de máxima actualidad, la potenciación de la eficacia de las formas de llevar a cabo las investigaciones y de reunir y analizar los datos o la ampliación y globalización de los temas investigados.

Mecanismos innovadores de financiación de la investigación. Se han creado becas de investigación innovadora para pequeñas empresas (Small Business Innovation Research Grants), contratos para el desarrollo de nuevos fármacos con investigadores o pequeñas empresas que no disponen de medios para iniciar los procedimientos formales de investigación que requieren las autoridades, becas para

\footnotetext{
Se puede encontrar información sobre este proyecto en http://www. edc.gsph.pitt.edu/reach/
}

financiar la realización de estudios piloto y la planificación de nuevos ensayos clínicos, y se han añadido componentes relacionados con la función cognitiva y la demencia a ensayos clínicos que ya estaban en curso, organizados por otros organismos de los NIH.

Mecanismos para aumentar la eficacia y eficiencia de las investigaciones. El programa de los Centros de Enfermedad de Alzheimer (Alzheimer's Disease Centers: ADC) promueve la investigación, la formación, la transferencia de tecnologías y los estudios multicéntricos sobre la EA, otras demencias y el envejecimiento cerebral normal. El Centro Nacional Coordinador para la Enfermedad de Alzheimer ( $\mathrm{Na}$ tional Alzheimer's Coordinating Center: NACC), creado en 1999, es un centro coordinador de datos que posibilita el análisis conjunto de los datos recogidos por los ADC y por otras fuentes, con el fin de facilitar la investigación sobre la EA. El Estudio Multicéntrico sobre la Enfermedad de Alzheimer (Alzheimer's Disease Cooperative Study: ADCS) fue diseñado por el NIA en 1991 como una estructura organizativa destinada a facilitar la cooperación entre distintos centros para investigar nuevos fármacos para el tratamiento de la EA y mejorar las pruebas diagnósticas utilizadas en los ensayos clínicos.

\section{APOYO DE OTROS INSTITUTOS DE LOS NIH}

El último capítulo de este informe da cuenta de las principales investigaciones realizadas en el campo de la EA bajo los auspicios de otros institutos de los NIH:

- El Instituto Nacional de Trastornos Neurológicos y Accidentes Cerebrovasculares (National Institute of Neurological Disorders and Stroke: NINDS), que está investigando los mecanismos moleculares de las lesiones neuronales y de la apoptosis.

- El Instituto Nacional de Salud Mental (National Institute of Mental Health: NIMH), que está contribuyendo a la investigación de las causas de la EA, de su evolución clínica, y de los tratamientos y servicios para los pacientes y quienes cuidan de ellos, con especial énfasis en el diagnóstico neurorradiológico y el tratamiento de los síntomas neuropsiquiátricos.

- El Instituto Nacional de Investigación en Enfermería (National Institute of Nursing Research: NINR), que apoya investigaciones sobre los aspectos bioconductuales de la EA y de otras demencias, y en especial de los problemas conductuales, físicos y funcionales, tales como la agitación, la agresividad o el mantenimiento de las actividades cotidianas. 
- El Instituto Nacional para el Alcoholismo y el Abuso de Alcohol (National Institute on Alcohol Abuse and Alcoholism: NIAAA), que está prestando especial atención al diagnóstico diferencial entre la EA y la demencia relacionada con el alcohol.

- El Instituto Nacional de Ciencias de la Salud Ambiental (National Institute of Environmental Health Sciences: NIEHS), que está investigando cómo los metales y otros contaminantes ambientales pueden afectar al cerebro y contribuir a la aparición de la EA.

- El Instituto Nacional de Salud Infantil y Desarrollo Humano (National Institute of Child Health and Human Development: NICHD), que está contribuyendo a la investigación de la EA, sobre todo a través de sus programas sobre la neurobio-logía del retraso mental y de los trastornos del desarrollo.

- El Instituto Nacional de Investigación del Genoma Humano (National Human Genome Research Institute: NHGRI), con una participación muy activa en las investigaciones sobre los aspectos genéticos de la enfermedad.

- El Centro Nacional de Medicina Complementaria y Alternativa (National Center for Complementary and Alternative Medicine: NCCAM), que está participando en investigaciones sobre el papel terapéutico de antioxidantes como la vitamina $\mathrm{E}$ o el extracto de Ginkgo biloba.

\section{PERSPECTIVAS FUTURAS}

Además de la continuación e intensificación de la colaboración entre los $\mathrm{NIH}$, otros organismos federales, las compañías farmacéuticas y otras organizaciones públicas y privadas, cabe destacar dos nuevas iniciativas lanzadas por los $\mathrm{NIH}$ :

- La Iniciativa de Prevención de la Enfermedad de Alzheimer (Alzheimer's Disease Prevention Initiative), que tiene varios frentes: estimulación de nuevos métodos de investigación epidemioló- gica y biológica básica; potenciación del descubrimiento y desarrollo de nuevos fármacos; mejora de los métodos para identificar precozmente a los individuos con riesgo de padecer EA; facilitación de la realización de ensayos clínicos de nuevos tratamientos, e investigación de nuevas estrategias, tanto farmacológicas como no farmacológicas, para tratar los trastornos conductuales de los pacientes con EA.

- La Iniciativa Presidencial sobre la Enfermedad de Alzheimer (President's Initiative on Alzheimer's Disease), anunciada en julio del 2000, a través de la cual se destinarán en los próximos 5 años 50 millones de dólares a la investigación sobre esta enfermedad.

\section{SYNOPSIS}

\section{Alzheimer's disease in the year 2000}

This document summarizes a report on the current state of knowledge of the etiology, diagnosis, and treatment of Alzheimer's disease and on the primary research activities carried out in these areas during the year 2000 under the auspices of the National Institute of Aging and other units of the National Institutes of Health (NIH) of the United States of America. In research on the etiology of the disease, key areas of attention have included amyloid, presenilins, apolipoprotein E, and other genetic ties; apoptosis; and protein aggregation in other neurodegenerative diseases. With respect to diagnosis, the progress that has been made is analyzed in the areas of neuroimaging and neuropsychological tests, clinical-pathological correlations, and the identification of biological disease markers, with the goal of being able to diagnose the disease before irreversible cognitive and functional deterioration takes place. Research on pharmacological treatment has been centered on three primary concerns: short-term maintenance of cognitive function, disease prevention, and treatment of behavioral symptoms. Another subject of great importance is support for those who provide care for patients. The report also deals with several areas related to research infrastructure and the contributions of other units of the NIH. 